\title{
GSM based Automatic Irrigation Control System for Efficient Use of Resources and Crop Planning by Using an Android Mobile
}

\author{
Pavithra D. S ${ }^{1}$, M. S .Srinath ${ }^{2}$ \\ ${ }^{\prime} P G$ Student, Department of ME, MCE, Hassan, India. \\ Associate Professor, Department of ME, MCE, Hassan, India.
}

\begin{abstract}
The greenhouse based modern agriculture industries are the recent requirement in every part of agriculture in India. In this technology, the humidity and temperature of plants are precisely controlled. Due to the variable atmospheric circumstances these conditions sometimes may vary from place to place in large farmhouse, which makes very difficult to maintain the uniformity at all the places in the farmhouse manually. It is observed that for the first time an android phone-control the Irrigation system, which could give the facilities of maintaining uniform environmental conditions are proposed. The Android Software Development Kit provides the tools and Application Programmable Interface necessary to begin developing applications on the Android platform using the Java programming language. Mobile phones have almost become an integral part of human life serving multiple needs of humans. This application makes use of the GPRS [General Packet Radio Service] feature of mobile phone as a solution for irrigation control system.

GSM (Global System for Mobile Communication) is used to inform the user about the exact field condition. The information is passed onto the user request in the form of SMS.
\end{abstract}

Keywords: GSM module (mobile), Android, SMS, automatic irrigation, microcontroller, Temperature sensor, soil moisture sensor, regulated power supply.

\section{Introduction}

Drip irrigation is artificial method of supplying water to the roots of the plant. It is also called micro irrigation. In past few years there is a rapid growth in this system. The user communicates with the centralized unit through SMS. The centralized unit communicates with the system through SMS which will be received by the GSM with the help of the SIM card. The GSM sends this data to ARM7which is also continuously receives the data from sensors in some form of codes. After processing, this data is displayed on the LCD. Thus in short whenever the system receives the activation command from the subscriber it checks all the field conditions and gives a detailed feedback to the user and waits for another activation command to start the motor. The motor is controlled by a simple manipulation in the internal structure of the starter. The starter coil is indirectly activated by means of a transistorized relay circuit. When the motor is started, a constant monitoring on soil moisture and water level is done \& once the soil moisture is reached to sufficient level the motor is automatically turned off \& a massage is send to subscriber that the motor is turned off. The water level indicator indicates three levels low, medium, high and also empty tank.

Shen etc. al (2007) introduced a GSM-SMS remote measurement and control system for greenhouse based on PC-based database system connected with base station. Base station is developed by using a microcontroller, GSM module, sensors and actuators. In practical operation, the central station receives and sends messages through GSM module. Criterion value of parameters to be measured in every base station is set by central station, and then in base stations parameters including the air temperature, the air humidity.

Indu etc. al (2013) mainly focuses on reviews in the field of remote monitoring and control, the technology used and their potential advantages. The paper proposes an innovative GSM/Bluetooth based remote controlled embedded system for irrigation. The system sets the irrigation time depending on the temperature and humidity reading from sensors and type of crop and can automatically irrigate the field when unattended. Information is exchanged between far end and designed system via SMS on GSM network. A Bluetooth module is also interfaced with the main microcontroller chip which eliminates the SMS charges when the user is within the limited range of few meters to the designated system. The system informs users about many conditions like status of electricity, dry running motor, increased temperature, water content in soil and smoke via SMS on GSM network or by Bluetooth.

The GSM based irrigation system [Fig.1] may offer users the flexibility to regulate and control the operations of their irrigation systems with little intervention to reduce runoff from over watering for improvement in crop yield. This enables users to take advantage of the globally deployed GSM networks with its low SMS service cost to use mobile phones and simple SMS commands to manage their irrigation system. It will be possible for users to use SMS to monitor directly the conditions of their farmland, schedule the water 
needs of crops, automatically control watering, and set control operational conditions in accordance with the water needs of crops. This will help minimize overwatering and crop production cost.

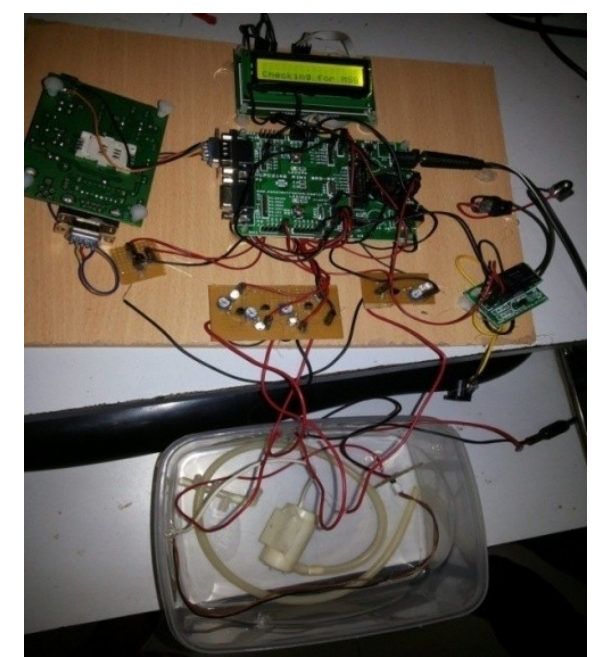

Fig.1: GSM based automatic irrigation control system by using an android mobile

Further, it will help users to take advantage of the prevailing GSM networks to provide value added services. The pump switching system was tested for functionality using a simple code to perform on-and-off operation of the LED. The functionality of the GSM was tested by connecting it to the microcontroller board which was programmed to turn on-and-off an LED using SMS from a mobile phone. The major objectives of the present work are,

- The system supports water management decision, which determines the controlling time for the process and monitoring the whole system through GSM module

- The system continuously monitors the water level in the tank and provide accurate amount of water required to the plant or tree (crop).

- The system checks the temperature, humidity and dew point so as to forecast the weather condition.

- Low cost and effective with less power consumption using sensors for remote monitoring and controlling devices which are controlled via SMS using a GSM using android mobile.

\section{Android Architecture}

Android is a mobile operating system that is based on a modified version of Linux. It was originally developed by a startup of the same name, Android, Inc. In 2005, as part of its strategy to enter the mobile space, Google purchased Android and took over its development work (as well as its development team).The following Fig.2 shows the major components of the Android operating system. The main advantage of adopting Android is that it offers a unified approach to application development and their applications should be able to run on numerous different devices, as long as the devices are powered using Android

Applications: These are applications written in Java. Some of basic applications include calendar, email client, SMS program, maps, making phone calls, accessing the Web browser, accessing your contacts list and others.

Application Framework: This is the skeleton or framework which all android developers have to follow. The developers can access all framework APIs an manage phone's basic functions like resource allocation, switching between processes or programs, telephone applications, and keeping track of the phone's physical location.

GSM: The Global System for Mobile Communication is a standard set developed by the European Telecommunications Standards Institute (ETSI) to describe technologies (2G and $3 \mathrm{G})$.

Libraries: This layer consists of Android libraries written in $\mathrm{C}, \mathrm{C}++$, and used by various systems. These libraries tell the device how to handle different kinds of data and are exposed to Android developers via Android Application framework. Some of these libraries includes media, graphics, 3D, SQL, web browser library etc. The Android runtime layer which includes set of core java libraries and DVM (Dalvik Virtual Machine) is also located in same layer. 
Runtime Android: This layer includes set of base libraries that are required for java libraries. Every Android application gets its own instance of DVM. Dalvik has been written so that a device can run multiple VMs efficiently and it executes files in executable (.Dex) optimized for minimum memory.

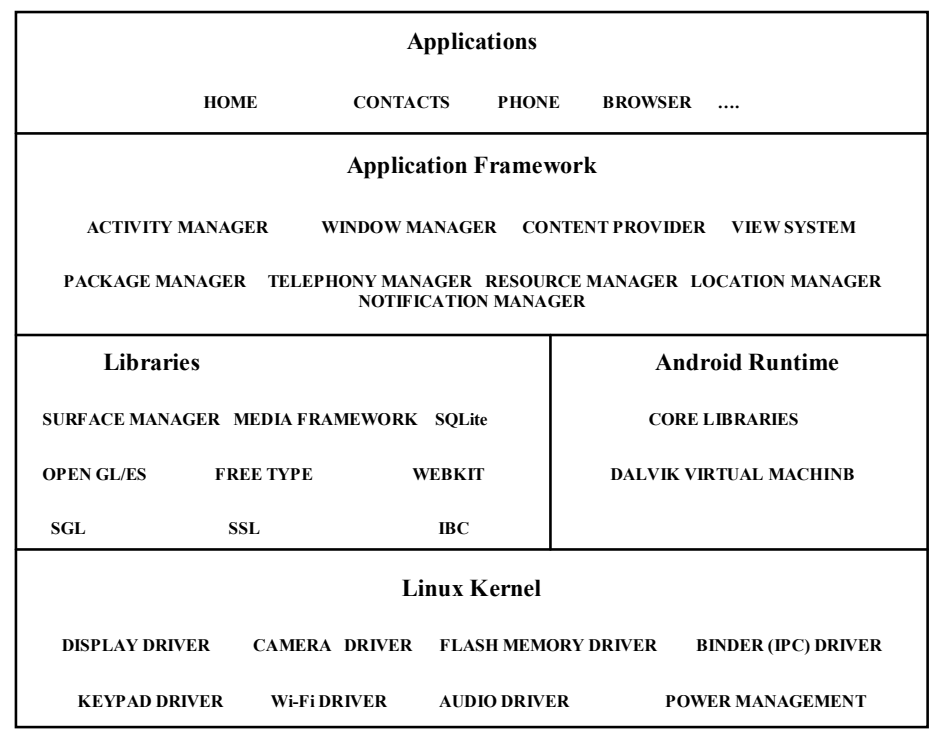

Fig.2: Android Architecture

Kernel Linux: This layer includes Android's memory management programs, security settings, power management software and several drivers for hardware, file system access, networking and inter-processcommunication. The kernel also acts as an abstraction layer between hardware and the rest of the software stack.

\section{GSM Based Irrigation Control System}

The connections between the two mobiles are done using GSM. The GSM module and microcontroller are connected using UART (universal asynchronous receiver / transmitter). When the moisture sensor senses the low moisture content of the soil, it gives a signal to the microcontroller. The microcontroller then gives a signal to the called mobile (which is kept in the auto answering mode). The called mobile activates the buzzer.

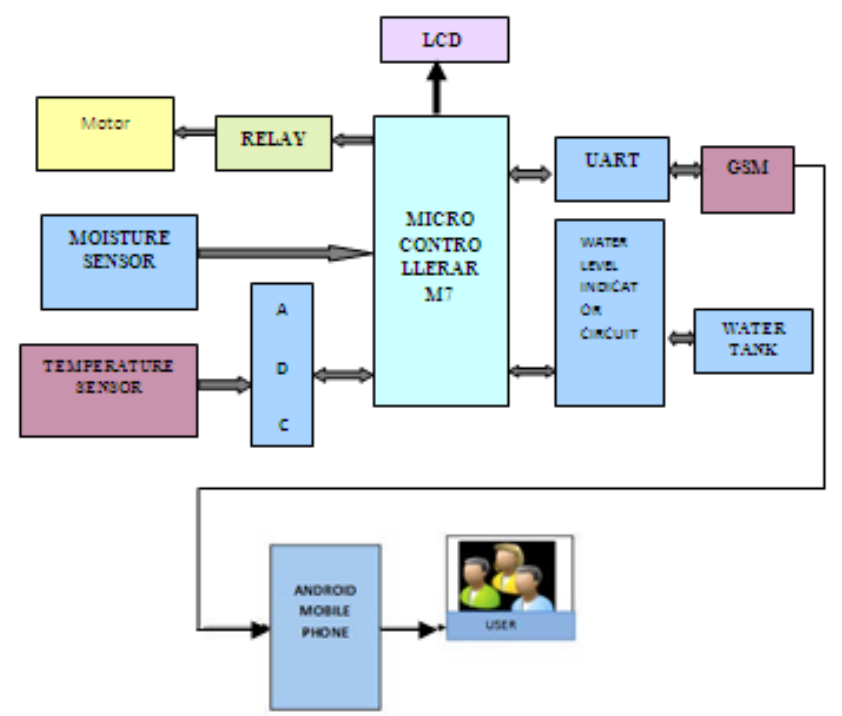

Fig.3: Block Diagram of the Irrigation Control System

Therefore when calling mobile calls, that buzzer is heard indicating the valve needs to be open. By pressing the button in the called function, the signal is given back to the microcontroller. The microcontroller gives signal to the valves which causes it to get open. The water is given to the root of the plant drop by drop, and when the moisture content becomes sufficient, the sensor senses this and gives back the signal to the 
microcontroller and the buzzer becomes off. Then by pressing the button in the calling function again, the valve is made off. The power supply needed by the controlling system is $+5 \mathrm{~V}$. The entire unit is as shown in Fig.3.

An UART is responsible for performing the main task in serial communications with computers. The device changes incoming parallel information to serial data which can be sent on a communication line. A second UART can be used to receive the information.

The UART performs all the tasks, timing, parity checking, etc. needed for the communication. The only extra devices attached are line driver chips capable of transforming the TTL level signals to line voltages and vice versa. The Microcontroller ARM7 structure is a general purpose 32-bit microprocessor, which offers high performance and very low power consumption. The ARM architecture is based on Reduced Instruction Set Computer (RISC) principles, and the instruction set and related decode mechanism are much simpler than those of micro programmed Complex Instruction Set Computers. Can be used to control water flow

\section{Structure Of GSM Module}

At present the GSM module is used for Remote Control activities such as Gate Control, Temperature Control etc. GSM/GPRS module consists of a GSM/GPRS modem assembled together with power supply circuit and communication interfaces (like RS-232, USB) for computer.

The MODEM is the soul of such modules. They generate, transmit or decode data from a cellular network, for establishing communication between the cellular network and the computer. These are manufactured for specific cellular network (GSM/UMTS/CDMA) or specific cellular data standard (GSM/UMTS/GPRS/EDGE/HSDPA) or technology (GPS/SIM). They use serial communication to interface with the user and need Hayes compatible AT (Attention) commands for communication with the computer (any microprocessor or microcontroller system).

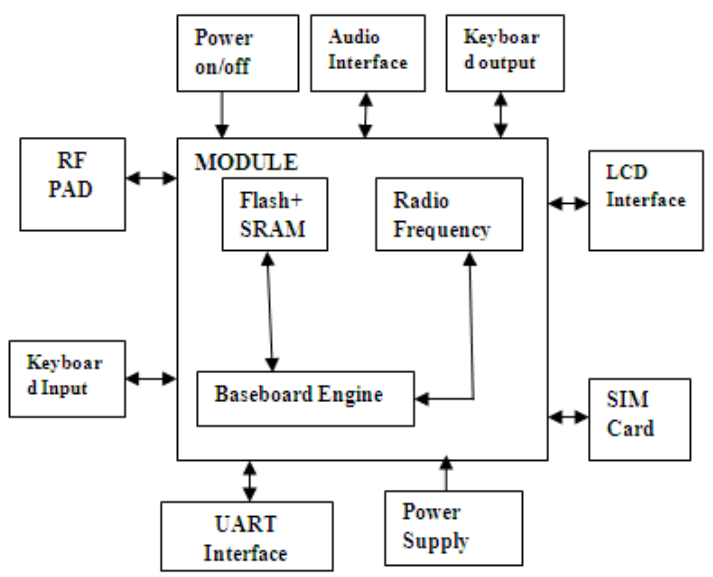

Fig.4: GSM module structure

An RS-232 port was once a standard feature of a personal computer for connections to modems, printers, mice, data storage, un-interruptible power supplies, and other peripheral devices. However, the limited transmission speed, relatively large voltage swing, and large standard connectors motivated development of the universal serial bus which has displaced RS-232 from most of its peripheral interface roles. Many modern personal computers have no RS-232 ports and must use an external converter to connect to older peripherals. Some RS-232 devices are still found especially in industrial machines or scientific instruments.

\section{Liquid/moisture sensor and precision centigrade temperature sensors}

Detects presence of liquid or moisture between two wire leads and gives active High output. The exposed wire is porous; therefore it allows transmission of water vapors into the sensor. These exposed areas are engineered very thinly. Therefore the sensor responds very rapidly to changes in applied moisture, both when being dried (on process start-up) and when called into action if there is moisture ingress into a process.

These are the types of sensors mainly used for,

-Interfacing with Microcontroller to detect liquid levels.

-Moisture detection for automatic watering of plants.

-Liquid level detection by putting multiple probes at each liquid level. 


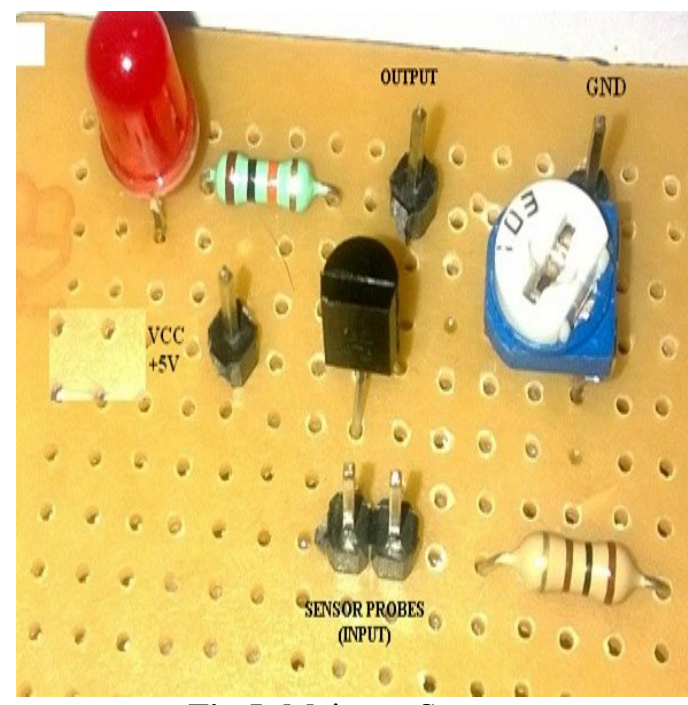

Fig.5: Moisture Sensor

Another important feature of this circuit is induction of Precision Centigrade Temperature Sensors. The LM35 is an integrated circuit sensor that can be used to measure temperature with an electrical output proportional to the temperature (in ${ }^{\circ} \mathrm{C}$ ). With an LM35, temperature can be measured more accurately than using a thermostat. The sensor circuitry is sealed and not subject to oxidation. The LM35 generates a higher output voltage than thermocouples and may not require the output voltage be amplified as shown in the Fig.6.

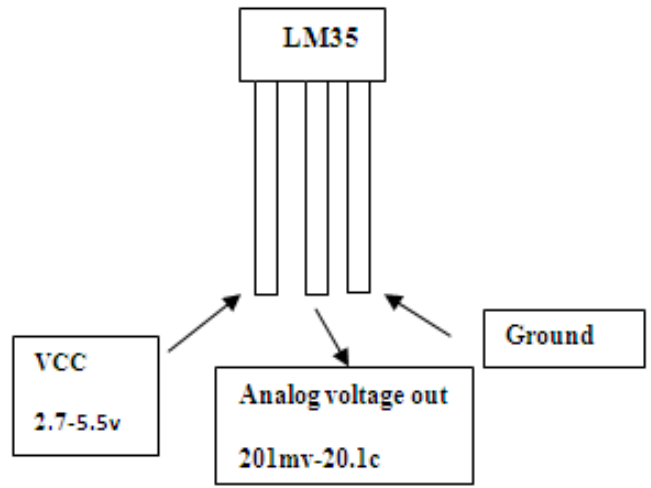

Fig.6: Precision Centigrade Temperature Sensors

\section{Water level indicator}

The model consists of a series of tanks arranged one below the other. The volume of the tanks is in descending order. Water flows from the top tank through outlets at the bottom. Three tanks or tropic levels chosen for the model is the optimum number required to analyze the effect of top down and bottom up controls. Each tank has two outlets, outlet A and outlet B. Each outlet has the water flow through it regulated by means of valves. These valves are controlled by floats in the tanks.

The system used microcontroller to automate the process of water pumping in an over-head tank storage system and has the ability to detect the level of water in a tank, switch on/off the pump accordingly and display the status on an LCD screen. 


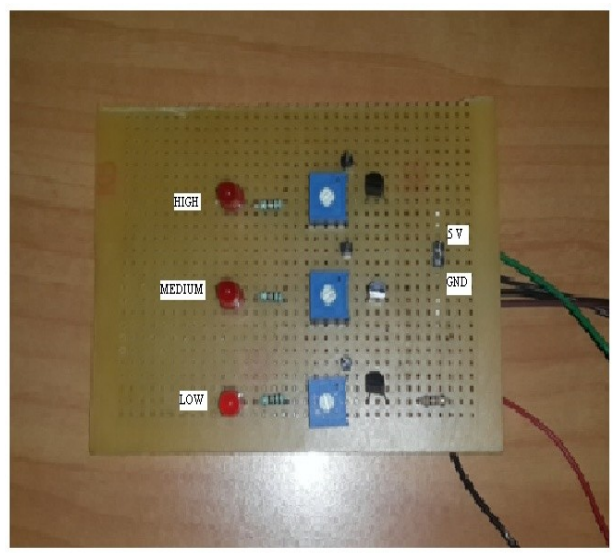

Fig7: Water level indicator

\section{Watering Times}

Times and intervals for watering differ according to the type of plant. The most important factor to remember is the depth of the root zone and soil composition. The deeper the roots and the finer the soil, the longer the watering time must be, then the frequency of watering will be reduced.

\section{Android Software development kit}

\section{Software Implementations}

Android software development is the process by which new applications are created for the Android operating system. Applications are usually developed in the Java programming language using the Android Software Development Kit. The Android software development kit (SDK) includes a comprehensive set of development tools. These include a debugger, libraries, a handset emulator based on QEMU, documentation, sample code, and tutorials. The SDK also supports older versions of the Android platform in case developers wish to target their applications at older devices. Development tools are downloadable components, so after one has downloaded the latest version and platform, older platforms and tools can also be downloaded for compatibility testing. Android applications are packaged in.apk format and stored under/data/app folder on the Android OS (the folder is accessible only to the root user for security reasons). APK package contains.dex files (compiled byte code files called Dalvik executable), resource files, etc...

\section{Conclusions}

Irrigation has been the backbone of human civilization since man has started agriculture. As the generation evolved, man developed many methods of irrigation to supply water to the land. In the present scenario on conservation of water is of high importance. Present work is attempts to save the natural resources available for human kind. By continuously monitoring the status of the soil, we can control the flow of water and thereby reduce the wastage.

By knowing the status of moisture and temperature through GSM with the use of moisture and temperature sensors, water flow can be controlled by just sending a message from our mobile.

Conservation of water and labor: Since the systems are automatic, they do not require continuous monitoring by labor.

System and operational flexibility: As desired, any valve can be controlled along with the pump and increases the efficiency of water use. If water is stored in tanks at irrigation lands, one can get the status of the status of the water level, temperature sensor and moisture content in soil through SMS generator by microcontroller present at the irrigation land.

The system has an incorporated Bluetooth for remote monitoring which reduces the problem of range with GSM network and saves SMS cost for the farmer. The smoke sensors used to send emergency information to user incase of fire in field or burning of motor. The design is low power, low cost, small size, robust and highly versatile.

Thus, this system avoids over irrigation, under irrigation, top soil erosion and reduce the wastage of water. The main advantage is that the system's action can be changed according to the situation (crops, weather conditions, soil etc.). By implementing this system, agricultural, horticultural lands, parks, gardens, golf courses can be irrigated. Thus, this system is cheaper and efficient when compared to other type of automation system.In large scale applications, high sensitivity sensors can be implemented for large areas of agricultural lands. A stand by battery or solar cells can be implemented which comes into use in case of power cuts. A secondary pump can be used in case of failure of the pump. 


\section{References}

[1]. Kay, M., "Smallholder irrigation technology: Prospects for sub-Saharan Africa" International Program for Technology and Research in Irrigation and Drainage, FAO, Rome, 2001, pp. 1-25.

[2]. N. Shah and I. Das, "Precision Irrigation Sensor Network Based Irrigation", a book on Problems, Perspectives and Challenges of Agricultural Water Management, IIT Bombay, India, pp. 217-232, April 2008

[3]. Fangmeier, D. D., Garrot, D. J.,Mancino, C.F and Husman, S. H., "Automated irrigation systems using plant and soil sensors", American Society of Agricultural Engineers, ASAE Publication, 1990, pp. 533-537.

[4]. Benzekri, A., Meghriche, K., and Refoufi, L., PC-based automation of a multi-mode control for an irrigation system Proceedings of International symposium on industrial embedded systems, Lisbon, July 2007, pp. 310-315.

[5]. Shinghal, K., Noor, A., Srivastava, N., and Singh, R., Wireless sensor networks in agriculture for potato farming International Journal of Engineering, Science and Technology, Vol. 2, No. 8, 2010, pp. 3955-3963.

[6]. [56Gautam, I., and Reddy, S. R. N.,Innovative GSM-Bluetooth based remote controlled embedded system for irrigation, International Journal of Computer Applications, Vol. 47, No. 8, 2012, pp. 1.

[7]. Zhang, F., Yang, M., and Ying, H., The application of GSM communication in agricultural automation, Journal of Technology for Agriculture, Vol. 1, No. 1, 2004, pp. 39-41.

[8]. Shen Jin, Song Jingling, Han Qiuyan, Wang Shengde, and YangYan, School of Electric and Electronic Engineering, A Remote Measurement and Control System for Greenhouse Based on GSM-SMS, IEEE 8th International Conference on Electronic Measurement and Instrument, 2007,pp. 45-82

[9]. Webin Huang, Guanglong Wang, Research of Wireless Sensor Networks for an Intelligent Measurement System Based on ARM, pp.1074-1079, 2011.

[10]. Sezen SM, Yazar A, Irrigation Management on Yield And Quality Of Tomatoes Grown in different Soilless Media in Glasshouse, 41-48, 2010.

[11]. Daniel K.Fisher and HirutKebede, a Low Cost Microcontroller-Based System to Monitor Crop Temperature and Water Status, pp. $168-173,2010$. 